\title{
Managing Visitor and Patient Flow During the COVID-19 Pandemic: ExpectingU App
}

\author{
Frederic EHRLER ${ }^{\mathrm{a}, 1}$, Remy TROMPIER ${ }^{\mathrm{a}}$, Guerric MERLE ${ }^{\mathrm{a}}$, Sebastien ANDRE ${ }^{\mathrm{a}}$, \\ Florian MÜLLER ${ }^{\mathrm{a}}$, and Katherine BLONDON ${ }^{\mathrm{a}}$ \\ ${ }^{a}$ University Hospitals of Geneva, Switzerland
}

\begin{abstract}
Access to hospitals has been dramatically restricted during the COVID 19 pandemic. Indeed, due to the high risk of contamination by patients and by visitors, only essential visits and medical appointments have been authorized. Restricting hospital access to authorized visitors was an important logistic challenge. To deal with this challenge, our institution developed the ExpectingU app to facilitate patient authorization for medical appointments and for visitors to enter the hospital. This article analyzes different trends regarding medical appointments, visitors' invitations, support staff hired and COVID hospitalizations to demonstrate how the ExpectingU system has helped the hospital to maintain accessibility to the hospital. Results shows that our system has allowed us to maintain the hospital open for medical appointments and visits without creating bottlenecks.
\end{abstract}

Keywords. COVID, patient flow management

\section{Introduction}

Access to hospitals has been dramatically restricted during the COVID 19 pandemic $[2,5]$. Indeed, the extremes contagiousness of the disease drove the hospital to limit access to the hospital, both to protect the patients but also the visitors. Restricting access to the hospital has many consequences. First patients without visitors are isolated from their family during a very difficult moment of their life. This is even more dramatic for patients with severe conditions such as those in intensive care, who may die without being able to see their family. Family presence has been shown to be an important source of psychological stability for the patient, as well as a source of support for better recovery $[3,6]$. During the first wave of the pandemic all non-essential medical and surgical activity and all visits were postponed [1,4]; during the second wave, it was important to maintain a minimal activity and allow limited visitors to avoid worsening the condition of non-COVID patients.

Controlling the access to the hospital is a daunting task, indeed, as the University Hospitals of Geneva (HUG) are spread out in several buildings, and each of them has several entrances. For employees, access is controlled by their badges. For patients and visitors, it was necessary to identify who was authorized to enter. In order to deal with this challenge, several departments of the HUG collaborated to develop an innovative solution facilitating the access control.

\footnotetext{
${ }^{1}$ Corresponding Author, Frederic EHRLER, University Hospitals of Geneva, Gabrielle-Perret-Gentil,4 1205 Geneve, Switzerland; E-mail: Frederic.Ehrler@hcuge.ch.
} 
The solution named "ExpectingU" connects a visitor's invitation forms in the EHR as well as the institutional agenda with an entry control system based on the scan of QR codes send through SMS (Figure 1). This system covers four use cases: patient visitors, medical appointments, elective hospitalization and professional invitation. To invite a visitor, the care provider creates an appointment with the visitor's phone number in an EHR form. Following this action, the visitor receives an invitation by SMS on her mobile phone. This invitation includes a link to a QR code that can be scanned by the entrance staff. When the QR code is scanned, the system will check that the visitor has come at the right time and that the invitation hasn't been already used. For medical appointments, an SMS with a QR code is automatically triggered when setting up the appointment with the institutional calendar. It is also important to mention that the app allows to manage in a unique place the rule of access that have evolved all along the pandemic. It allowed caregivers to focus on their important duty without having them to worry about visiting policies (number of visitors, frequency and duration).

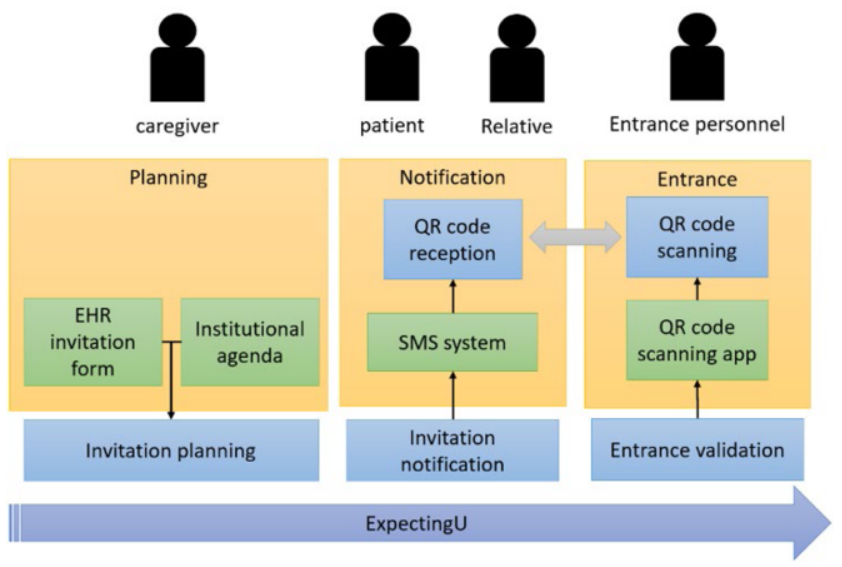

Figure 1. Process of the expectingU system

In this article we report the deployment of the solution and analyze its impact on attribution of admission personnel to control patient entry. We hypothesized that the Expecting $U$ app would help decrease the number of staff hired to manage visitors and outpatient flow.

\section{Method}

Usage logs were analyzed in order to understand the dynamics of the patient flow in the hospital during the COVID-19 period (March to December 2020). Four sources of information were queried: the use of the invitation form for visitors, the number of ambulatory medical appointments recovered from the institutional agenda, the number of full-time (FT) support staff hired to deal with the situation and the number of COVID hospitalizations at HUG. Each indicator value was normalized relatively to its highest values in the observation period and displayed conjointly with the other indicators in order to analyze the trends. 


\section{Result}

By looking at the number of COVID hospitalizations on the figure 2, we clearly see the two waves. The first one from March to April and the second one from October to December (although continuing certainly in 2021). The first peak reaches 9632 hospitalizations during April and the second peak reaches 13723 hospitalizations in November.

In normal conditions, there are about $45^{\prime} 000$ outpatient medical appointments every month. We see that at the beginning of the first wave, the number of appointments in the hospital dropped to $60 \%$ in March and then to $30 \%$ in April (11'200) of the usual appointment level. In May, medical activity increases progressively and reaches its usual level in June. During the second wave, starting in November, we have also a reduction of visits, which remains much less important than during the first wave with about $70 \%$ of medical appointments.

Table 1. Values of the different indicators in the observation period (March to December 2020)

\begin{tabular}{lrlrr}
\hline Month & Invitation Form & $\begin{array}{l}\text { Entrance } \\
\text { personnel }\end{array}$ & $\begin{array}{l}\text { Medical } \\
\text { Appointment }\end{array}$ & $\begin{array}{l}\text { COVID } \\
\text { Hospitalization }\end{array}$ \\
\hline March & 283 & 5 & 23588 & 3360 \\
April & 773 & 8 & 11294 & 9632 \\
Mai & 4920 & 5 & 25169 & 3543 \\
June & 11075 & 23 & 36140 & 104 \\
July & 9890 & 33 & 33762 & 164 \\
August & 10091 & 39 & 32554 & 400 \\
September & 9091 & 40 & 37063 & 538 \\
October & 8411 & 31 & 35784 & 4546 \\
November & 8457 & 106 & 28790 & 13723 \\
December & 8839 & 94 & 26899 & 2621 \\
\hline
\end{tabular}

Concerning the invitation forms for visitors, we see that almost no invitations were sent at the month of March and April: no visits were allowed initially, and the whole ExpectingU system was not yet in place. The system was deployed end of April. From May on, we observe a strong increase of invitation forms that reach its peak in June with 11075 invitations. After that the number of invitation forms sent remains stable around $80 \%$ of the maximum.

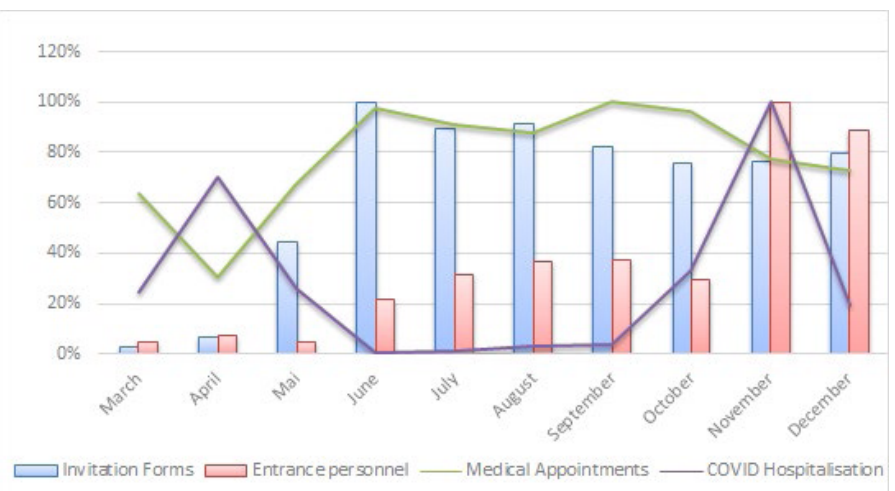

Figure 2. Evolution of the trends over time (all indicators are given relatively to the highest value un the period) 
Finally, for the support staff hired to manage all the hospital entrances on the different sites, until May the number of support staff was low with less than 8 people simultaneously in average. From June to October, this number significantly increase from 24 people in June to 40 in September. During the last wave starting in November, the number of support staff exceeded 100.

Weekly usage of the scan system by support staff at beginning of January shows clearly the scale of the system (figure 3). Each peak starts each day at 6 am until $7 \mathrm{pm}$ with a significant reduction at lunchtime. These peaks correspond clearly to the appointment and visit schedule. We also observe a clear reduction of scans during the weekend since no medical appointment are scheduled then. At its highest level, the scan system was used by about 24 FT staff to check nearly 2000 individuals per day $(30 \%$ were visitors and $70 \%$ were patients on average) at the various hospital entrances.

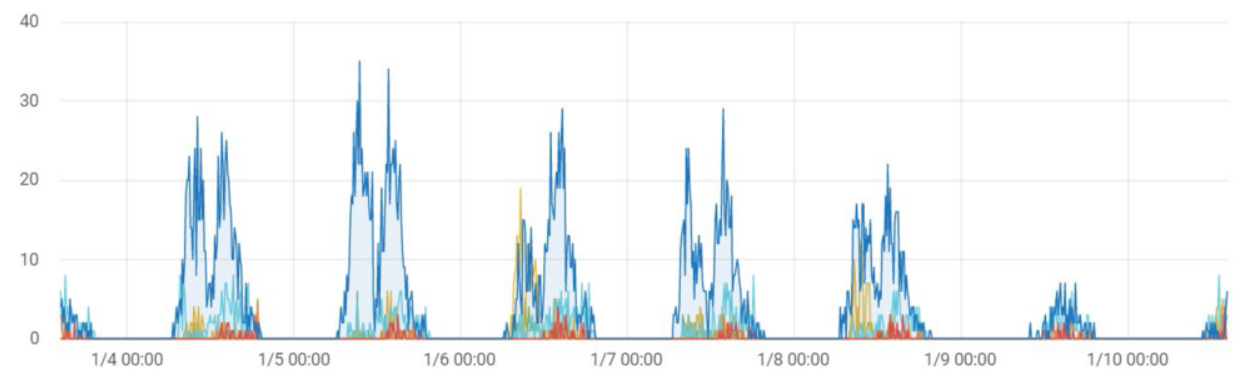

Figure 3. QR code scan in the second week of January 2021

\section{Discussion}

During the first wave, visitors were not allowed, and outpatient activity was suspended. At the end of May when the situation improved, visits to patients and outpatient appointments resumed progressively to a maximum of 3 visitors per patient/day and usual medical activity by the end of June. At that time, 24 FT support staff were hired to man all the various hospital entrances on the different sites. Before the ExpectingU app was implemented, patients needed to present their appointment invitation (sent by mail) or the text message reminder. For visitors, the admissions staff had to call the wards to confirm the permission to visit.

After implementing the app, the number of hospital staff required to verify hospital access did not change, but allowed more visitors with less wait lines. The nurses in the wards fill in a form for each patient and each visit, which sends a text message to the visitor with a QR code. Hospital staff use the ExpectingU app to scan the code and verify access. Visitors could also present the form for their next visit. Hospital staff positions remained at 24 FT positions during this time. Patient and visitor flow were much improved, as the app helped accelerate the verification process, and there was no wait line.

During the second wave, our hospital decided to maintain visits and out-patient appointments. Since ward unit doors were closed (badge access) 72 FT additional hospital staff were required to help unlock the doors for visitors, to reinforce COVID measures (hand disinfection, masks, as well as to assist with gown, gloves, and later on, visitors) and to limit the duration of visits. This additional pool of hospital staff also 
provided occasional support with the entrance access, during COVID-related absences for example. During the decreasing period of the second wave, these 72 FT decreased to $20 \mathrm{FT}$ as ward doors were unlocked, and COVID measures decreased. These residual 20 FT continue to help visitors and limit visit durations in the wards and are supplemental to the $24 \mathrm{FT}$ at the entrances.

Since the implementation of the app, there have been several changes in workflow for nursing, admission and support staff. First, nurses needed to fill in the form, and our data show that adoption of the form was rapid, with currently around 8500 forms per month. Finally, although the number of support staff positions did not decrease with the app, wait times at the admissions desk, patient satisfaction and ease of verification were a big improvement for all. There have been very few if any technical issues with the app; the main residual issues with the app are principally device use, or individual attitudes (refusal to submit to such procedures). Admissions officers continue to verify access for visitors who do not have phones, or for those who forget their permission/appointment form.

\section{Conclusion}

Maintaining an access for patients and visitors has been a real logistical challenge during the COVID pandemic. The constraint associated with access control to contain the infections has led many hospitals to restrict patient and visitor entry leading to a reduction of medical activity and the isolation of patients. By connecting existing systems of our EHR with a system to control patient flow, the HUG has managed to limit the impact of COVID on its visitor policies without creating bottlenecks. Although not evaluated in our study limiting the restriction on ambulatory appointment has potentially mitigated the economic impact of the pandemic. The solution has received a very positive feedback from all the patients even the oldest ones that were not familiar with digital solutions. It allows also to maintain a link between the patients and their families and act in the direction of a patient-centered model of care.

\section{References}

[1] Hübner M, Zingg T, Martin D, Eckert P, Demartines N. Surgery for non-Covid-19 patients during the pandemic. PLoS One. 2020 Oct 23;15(10):e0241331.

[2] Liu YA, Hsu YC, Lin MH, Chang HT, Chen TJ, Chou LF, Hwang SJ. Hospital visiting policies in the time of coronavirus disease 2019: A nationwide website survey in Taiwan. J Chin Med Assoc. 2020 Jun;83(6):566-570.

[3] Parsapour K, Kon AA, Dharmar M, McCarthy AK, Yang HH, Smith AC, Carpenter J, Sadorra CK, Farbstein AD, Hojman NM, Wold GL, Marcin JP. Connecting hospitalized patients with their families: Case series and commentary. Int. J. Telemed. Appl. 2011.

[4] Peloso A, Moeckli B, Oldani G, Triponez F, Toso C. Response of a European surgical department to the COVID-19 crisis. Swiss Med Wkly. 2020 Apr 9;150:w20241.

[5] Siddiqi H. To Suffer Alone: Hospital Visitation Policies During COVID-19. J Hosp Med. 2020 Nov;15(11):694-695

[6] Virani AK, Puls HT, Mitsos R, Longstaff H, Goldman RD, Lantos JD, Benefits and risks of visitor restrictions for hospitalized children during the COVID pandemic, Pediatrics. 2020; 146. 